\title{
WAVELET ANALYSIS OF THE MULTIVARIATE FRACTIONAL BROWNIAN MOTION
}

\author{
Jean-François Coeurjolly ${ }^{1,2}$, Pierre-Olivier Amblard ${ }^{2,3}$ \\ AND SOPHIE ACHARD ${ }^{2}$
}

\begin{abstract}
The work developed in the paper concerns the multivariate fractional Brownian motion $(\mathrm{mfBm})$ viewed through the lens of the wavelet transform. After recalling some basic properties on the $\mathrm{mfBm}$, we calculate the correlation structure of its wavelet transform. We particularly study the asymptotic behaviour of the correlation, showing that if the analyzing wavelet has a sufficient number of null first order moments, the decomposition eliminates any possible long-range (inter)dependence. The cross-spectral density is also considered in a second part. Its existence is proved and its evaluation is performed using a von Bahr-Essen like representation of the function $\operatorname{sign}(t)|t|^{\alpha}$. The behaviour of the cross-spectral density of the wavelet field at the zero frequency is also developed and confirms the results provided by the asymptotic analysis of the correlation.
\end{abstract}

Mathematics Subject Classification. 26A16, 28A80, 42C40.

Received December 7, 2011. Revised April 17, 2012.

\section{Motivations And OVERLOOK}

The fractional Brownian (fBm) motion developed by Mandelbrot and Van Ness [18] has been extensively studied as the archetypal model of fractal signals. Many extensions have been proposed, trying to keep the simplicity of its definition while modelling more complex phenomena. For example, time-dependent Hurst exponent or $d$-dimensional extensions have been introduced which have respectively led to the multifractional Brownian motion [19] and the fractional Brownian sheet [6]. Another extension consists in defining multivariate fractal processes. This extension is needed by many applications ranging from economy to physics, passing by biology and neuroscience $[1,2,5,15]$. In all these disciplines, many modern sensing approaches allow to measure instantaneously different variables from complex phenomena. The need of multivariate signals models is crucial in order to model and understand these phenomena.

A multivariate extension of the $\mathrm{fBm}$ has been proposed recently in a very general setting by Didier and Pipiras [10] with the help of operator self-similarity. The operator fractional Brownian motion is an operator selfsimilar Gaussian process with stationary increments. When the operator is diagonal, it is called the multivariate fractional Brownian motion $(\mathrm{mfBm})$. We have particularly studied this diagonal case [4], elaborating on the work

\footnotetext{
Keywords and phrases. Multivariate fractional Brownian motion, wavelet analysis, cross-correlation, cross-spectrum.

1 Laboratory Jean Kuntzmann, Grenoble University, France.

2 GIPSAlab/CNRS, Grenoble University, France.

3 Dept. of Mathematics\&Statistics, University of Melbourne, Australia. Jean-Francois.Coeurjolly@upmf-grenoble.fr
} 
of Lavancier et al. [17]. In these works, the correlation structure of the mfBm has been studied. The increments process has also been studied, and we have exhibited its correlation and spectral structures, showing the possible existence of long-range dependence in correlation as well as in cross-correlation between components.

In this paper, we pursue the study by wavelet analyzing the multivariate process. Wavelet transforms or decomposition provide a regularized differentiation of the processes, have a filter bank structure in perfect adequacy with $1 / f$ type of spectral behaviour, may eliminate long-range dependence properties if the analyzing wavelet is properly chosen. Therefore, it is now well-accepted that wavelet analysis is the appropriate framework to deal with monovariate fractal signals with stationary increments $[7,11-13,20,23]$ and in particular the appropriate framework to estimate efficiently the Hurst exponent (or the long-memory parameter in a semiparametric framework). Henceforth, establishing theoretical properties of the wavelet transform of the $\mathrm{mfBm}$ is of major interest in the perspective to estimate the parameters of this model (in particular the Hurst exponents and the correlation between the components). Based on the results of the present paper, the identification of the mfBm has been investigated in Amblard and Coeurjolly [3].

We thus concentrate on the correlation and on the spectral structure of the wavelet transform of the mfBm. The principal result of the paper is the explicit form of the second order statistics of this multivariate Gaussian random field. We study the asymptotic behaviour of the cross-correlation function of the wavelet, and this allows us to prove that choosing a wavelet with at least two null first order moments eliminates any possible long-range dependence in the correlations. We prove the existence and calculate the cross-spectral density of the wavelet field. We thus extend the result of Kato and Masry [16] providing the existence of the spectral density of the wavelet transform of the $\mathrm{fBm}$. The proof uses a generalization of the von Bahr-Essen representation of $|t|^{\alpha}$ (used by Kato and Masry [16]) to the function $\operatorname{sign}(t)|t|^{\alpha}$. We also provide the behaviour of the density at the zero frequency, corroborating the asymptotic result obtained in the time domain. We stress on the fact that our asymptotic results do not impose that the wavelet is a real function, nor that it has a compact support.

The paper is organized as follows. In order to have a self-contained exposition, we recall some basics definition and results on the $\mathrm{mfBm}$ in Section 2. In Section 3, we set the wavelet analysis and look at some self-similarity properties inherited from the process. The full correlation structure and the spectral counterpart are also presented in Section 3. A conclusion is presented in Section 4. The technical proofs and the needed generalized von Bahr-Essen representation are postponed into the Appendix.

\section{Some facts on the multivariate fractional Brownian motion}

The $p$ dimensional multivariate fractional Brownian motion $(\mathrm{mfBm}) x(t)$ is defined as the only Gaussian process having stationary increments and having components jointly self-similar with parameters $\left(H_{1}, \ldots, H_{p}\right) \in$ $(0,1)^{p}$. The self-similarity property can be stated as follows: for any real $\lambda>0, x(\lambda t) \stackrel{\text { fidi }}{=} \lambda^{H} x(t)$ where $H=\operatorname{diag}\left(H_{1}, \ldots, H_{p}\right)$ and $\lambda^{H}$ is intended in the matrix sense. The notation $\stackrel{\text { fidi }}{=}$ stands for equality of all the finite-dimensional probability distributions.

The cross-covariance structure induced by the multivariate self-similarity property and the stationarity of the increments has been first studied by Lavancier et al. [17], Theorem 2.1, without having recourse to the Gaussian assumption. Amblard et al. [4] have parameterized this covariance structure in a more simple way as follows.

Proposition 2.1 ([4], Prop. 3). Let $j, k \in\{1, \ldots, p\}, j \neq k$, then there exist $\sigma_{j}>0,\left(\rho_{j k}, \eta_{j k}\right) \in[-1,1] \times \mathbb{R}$ satisfying $\rho_{j k}=\rho_{k j}$ and $\eta_{j k}=-\eta_{k j}$, such that

$$
r_{j k}(s, t):=\mathbb{E}\left[x_{j}(s) x_{k}(t)\right]=\frac{\sigma_{j} \sigma_{k}}{2}\left\{w_{j k}(-s)+w_{j k}(t)-w_{j k}(t-s)\right\},
$$

where the function $w_{j k}(h)$ is defined by

$$
w_{j k}(h)= \begin{cases}\left(\rho_{j k}-\eta_{j k} \operatorname{sign}(h)\right)|h|^{H_{j}+H_{k}} & \text { if } H_{j}+H_{k} \neq 1, \\ \rho_{j k}|h|+\eta_{j k} h \log |h| & \text { if } H_{j}+H_{k}=1 .\end{cases}
$$



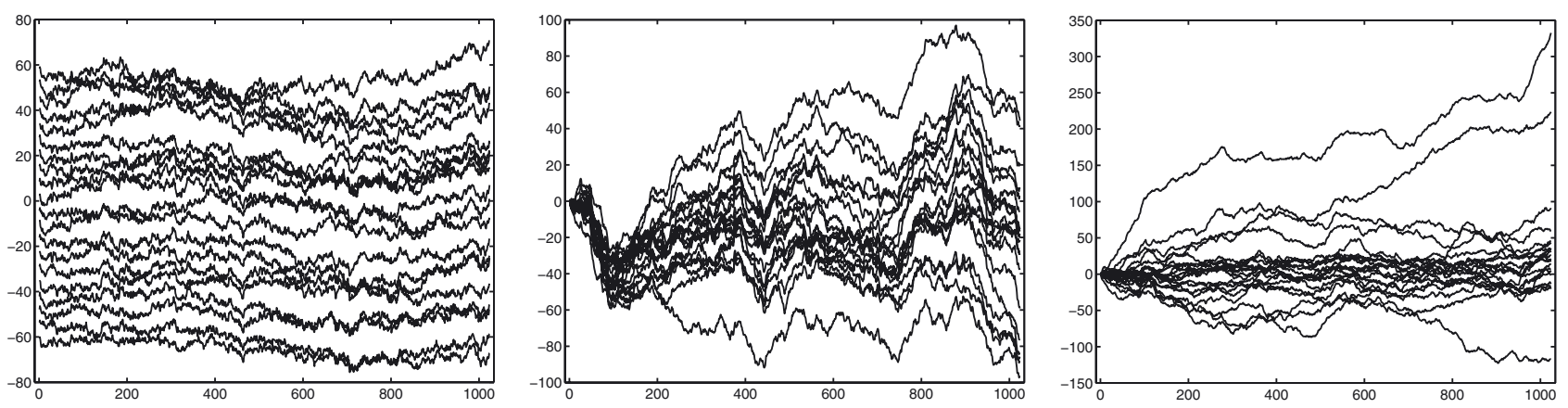

FiguRE 1. Examples of discretized sample paths of a time reversible $\left(\eta_{j k}=0\right) \mathrm{mfBm}$ of length $n=1024$, with $p=20$ components. The Hurst exponents are equally spaced in $[0.3,0.4]$ (left plot), $[0.6,0.7]$ (middle plot) and $[0.4,0.8]$ (right plot). The correlation parameters are set to 0.7 (left and middle plot) and to 0.3 (right plot). The components are shifted artificially in the left plot for the sake of visibility.

This result is also valid in the case $j=k$ when setting $\rho_{j j}=1$ and $\eta_{j j}=0$ in (2); we thus recover the covariance structure of a monovariate $\mathrm{fBm}$. The parameter $\sigma_{j}^{2}$ is the variance of a $\mathrm{fBm}$ at time $1, \operatorname{Var}\left(x_{j}(1)\right)$, whereas $\rho_{j k}$ represents the instantaneous correlation between components $j$ and $k$ at time 1 , i.e. $\mathbb{E}\left[x_{j}(1) x_{k}(1)\right]$. The antisymmetric parameter $\eta_{j k}$ is related to the time-reversibility property of the multivariate process. Indeed, the $\mathrm{mfBm}$ is time reversible, i.e. $x(t)=x(-t)$ in distribution for every $t$, if and only if $\eta_{j k}=0$ for all $j, k$ [4].

To ensure that the matrix given by (1) is the cross-covariance matrix of a process, the constraints imposed on $\rho_{j k}$ and $\eta_{j k}$ are not sufficient. A necessary and sufficient condition, proved by Amblard et al. [4], corresponds to the positive-definiteness of the Hermitian matrix with entries $\Gamma\left(H_{j}+H_{k}+1\right) \times \xi_{j k}$ where $\xi_{j k}$ is defined by

$$
\xi_{j k}= \begin{cases}\rho_{j k} \sin \left(\frac{\pi}{2}\left(H_{j}+H_{k}\right)\right)-\mathbf{i} \eta_{j k} \cos \left(\frac{\pi}{2}\left(H_{j}+H_{k}\right)\right) & \text { if } H_{j}+H_{k} \neq 1 \\ \rho_{j k}-\mathbf{i} \frac{\pi}{2} \eta_{j k} & \text { if } H_{j}+H_{k}=1\end{cases}
$$

where $\mathbf{i}=\sqrt{-1}$. For example, when $p=2$ and $\eta_{j k}=0$ no condition is required for the correlation $\rho_{12}$ when $H_{1}=H_{2}$, and when $H_{1}=0.1$ and $H_{2}=0.8$ the correlation $\rho_{12}$ cannot exceed 0.514 , see [4] for more discussion. The problem of simulation of such a process has been investigated in Amblard et al. [4] using the algorithm developed by Chan and Wood [8]. Figure 1 presents some examples in order to illustrate the process.

The covariance structure of the increment process (at lag 1) can be easily deduced from (1). When $j=k$, we obviously recover the covariance of the fractional Gaussian noise and the classical property that this process is short-memory when $H_{j} \leq 1 / 2$ and long-memory when $H_{j}>1 / 2$. In the multivariate case, long-range (interdependence) may also appear in the cross-covariance. Indeed Amblard et al. [4] proved that for all $j \neq k$, the cross-covariance behaves asymptotically as $|h|^{H_{j}+H_{k}-2}$ (up to a constant) meaning that the long-memory property arises as soon as $H_{j}+H_{k} \geq 1$ which can appear in three different situations: $H_{j}=H_{k}=1 / 2$, $H_{j}<1 / 2$ and $H_{k}>1-H_{j}$ or $H_{j}>1 / 2$ and $H_{k}>1 / 2$. In those cases, some troubles may appear when it comes to infer parameters of the models from data. Indeed, long-range dependence may lead to very slow convergence of estimators. As already observed in many works, e.g. $[7,9,13,21]$, having recourse to wavelet types of transformation is an elegant way to overcome the problem. Indeed, using wavelet types of transformation with a correctly chosen filter allows to extract the stationary part from the fBm and allows to "whiten" the increments. We describe such an approach in the following section. 


\section{WAVElet Analysis of The mFBM}

The use of wavelet analysis in the understanding of the monovariate fractional Brownian motion, and more generally for the study of fractal signals, goes back to the early works of Flandrin [12,13], Tewfik and Kim [20], Wornell [23] to cite some but a few.

The aim is now to analyze the multivariate fractional Brownian motion through the lens of the wavelet transform. We use the continuous wavelet transform here, but a similar analysis could be performed in the multiresolution framework using orthonormal wavelet bases. We will consider complex valued wavelets, not necessarily in the Hardy class, not necessarily with compact support. The hypothesis we impose on the wavelets will be detailed when needed.

\subsection{Definition and stationarity}

Let $\psi$ be a complex wavelet function, let $a>0$ and $b \in \mathbb{R}$ and consider $\psi_{a b}()=.a^{-1 / 2} \psi((.-b) / a)$. Let

$$
d_{a, b}^{j}:=\left\langle x_{j} \mid \psi_{a, b}\right\rangle_{L^{2}}=\int_{\mathbb{R}} x_{j}(t) \overline{\psi_{a, b}}(t) \mathrm{d} t
$$

the wavelet transform of the $j$ th component of a multivariate fractional Brownian motion. $\bar{\psi}$ denotes the complex conjugate of $\psi$. In this section, we assume that conditions [C1] and [C2(2)] are satisfied, where:

[C1] Admissibility condition: $\psi(t) \in L^{2}$ and $|\widehat{\psi}(\omega)|^{2} /|\omega| \in L^{1}$, where $\widehat{\psi}$ is the Fourier transform of $\psi$.

$[\mathrm{C} 2(\mathrm{~K})] t^{m} \psi(t) \in L^{1}$ for $m=0,1, \ldots, K$.

Condition [C1] ensures that $\widehat{\psi}(0)=0$ and that $\int_{\mathbb{R}} \psi(t) \mathrm{d} t=0$. We note, as Kato and Masry [16], that under condition $[\mathrm{C} 2(1)]$, the integral (4) is well-defined as a sample path integral and is a second-order random variable. This follows, since under $[\mathrm{C} 2(1)]$ we have $\int_{\mathbb{R}}|s|^{H}\left|\psi_{a, b}(s)\right| \mathrm{d} s<+\infty, \forall H \in(0,1)$.

The aim of this section is to focus on the correlation between the wavelet transforms (at different scales and different times) of two components $j$ and $k$ of the multivariate fractional Brownian motion. The wavelet transform is a random field. It is clearly zero mean and Gaussian. We have for $a_{1}, a_{2}>0$ and $b, h \in \mathbb{R}$

$$
E\left[d_{a_{1}, b+h}^{j} \overline{d_{a_{2}, b}^{k}}\right]=\int_{\mathbb{R}^{2}} r_{j k}\left(t_{1}, t_{2}\right) \overline{\psi_{a_{1}, b+h}}\left(t_{1}\right) \psi_{a_{2}, b}\left(t_{2}\right) \mathrm{d} t_{1} \mathrm{~d} t_{2}
$$

Under [C1], and from (2) the last expression reduces to

$$
E\left[d_{a_{1}, b+h}^{j} \overline{d_{a_{2}, b}^{k}}\right]=-\frac{\sigma_{j} \sigma_{k}}{2} \int_{\mathbb{R}^{2}} w_{j k}\left(t_{2}-t_{1}\right) \overline{\psi_{a_{1}, b+h}}\left(t_{1}\right) \psi_{a_{2}, b}\left(t_{2}\right) \mathrm{d} t_{1} \mathrm{~d} t_{2} .
$$

Let $\Gamma_{\psi}(v):=\int_{\mathbb{R}} \psi_{a_{1}, b+h}(u) \overline{\psi_{a_{2}, b}}(u+v) d u$ be the correlation function between the two wavelets $\psi_{a_{1}, b+h}$ and $\psi_{a_{2}, b}$. Then we have

$$
E\left[d_{a_{1}, b+h}^{j} \overline{d_{a_{2}, b}^{k}}\right]=-\frac{\sigma_{j} \sigma_{k}}{2} \int_{\mathbb{R}} w_{j k}(v) \overline{\Gamma_{\psi}}(v) \mathrm{d} v .
$$

Note that $[\mathrm{C} 2(2)]$ implies that for all the values of $H_{j}$ and $H_{k}, \int_{\mathbb{R}}\left|w_{j k}(v)\right|\left|\Gamma_{\psi}(v)\right| \mathrm{d} v<+\infty$. With two changes of variables, this may also be rewritten as

$$
E\left[d_{a_{1}, b+h}^{j} \overline{d_{a_{2}, b}^{k}}\right]=-\frac{\sigma_{j} \sigma_{k}}{2} \sqrt{a_{1} a_{2}} \times \int_{\mathbb{R}^{2}} w_{j k}\left(a_{2} t_{2}-a_{1} t_{1}-h\right) \bar{\psi}\left(t_{1}\right) \psi\left(t_{2}\right) \mathrm{d} t_{1} \mathrm{~d} t_{2} .
$$

If we interpret for fixed parameters $a_{1}$ and $a_{2}$, the quantity $E\left[d_{a_{2}, b+h}^{j} \overline{d_{a_{2}, b}^{k}}\right]$ as the cross-correlation between two signals, we observe that it depends only on the difference between the times at which it is evaluated (i.e. $h$ ). With the fact that the wavelet transform is a zero mean and Gaussian field, we conclude that $d_{a_{1}, \text {, and }}^{j} d_{a_{2}, \text {,. }}^{k}$ are jointly stationary signals. 


\subsection{Self-similarity type property of the cross-wavelet transform}

The variance of the wavelet transform at similar scales for the fractional Brownian motion with Hurst parameter $H$ exhibits a self-similarity property. Indeed, it is proved in Flandrin [12] for example that for all $b$

$$
\operatorname{Var}\left(d_{a, b}^{j}\right)=a^{2 H+1} \times\left(-\frac{\sigma^{2}}{2} \int_{\mathbb{R}^{2}}\left|t_{2}-t_{1}\right|^{2 H} \bar{\psi}\left(t_{1}\right) \psi\left(t_{2}\right) \mathrm{d} t_{1} \mathrm{~d} t_{2}\right) .
$$

We note here that the same behaviour holds for the cross-wavelet variance.

Proposition 3.1. Under the assumptions [C1] and [C2(2)], let $h=0$ and fix $a_{1}=a_{2}=a>0$. Then for all $b \in \mathbb{R}$,

$$
E\left[d_{a, b}^{j} \overline{d_{a, b}^{k}}\right]=a^{H_{j}+H_{k}+1}\left(-\frac{\sigma_{j} \sigma_{k}}{2} z_{j k}\right) \quad \text { and } \quad \operatorname{Corr}\left[d_{a, b}^{j}, d_{a, b}^{k}\right]=\frac{z_{j k}}{\sqrt{z_{j j} z_{k k}}}
$$

where $z_{j k}:=\int_{\mathbb{R}^{2}} w_{j k}\left(t_{2}-t_{1}\right) \bar{\psi}\left(t_{1}\right) \psi\left(t_{2}\right) \mathrm{d} t_{1} \mathrm{~d} t_{2}$.

Proof. Consider equation (6). The result is obvious when $H_{j}+H_{k} \neq 1$ since for any $a>0, w_{j k}(a v)=$ $a^{H_{j}+H_{k}} w_{j k}(v)$. Now, when $H_{j}+H_{k}=1$, the result comes from Condition [C1] ensuring that $\int_{\mathbb{R}^{2}} \eta_{j k} \times a\left(t_{2}-\right.$ $\left.t_{1}\right) \log (a) \bar{\psi}\left(t_{1}\right) \psi\left(t_{2}\right) \mathrm{d} t_{1} \mathrm{~d} t_{2}=0$.

\subsection{Cross-correlation structure of the wavelet transform of the $\mathrm{mfBm}$}

For fixed scales, $a_{1}, a_{2}$, we now specify the behaviour of the cross-wavelet covariance (or correlation) as $|h| \rightarrow+\infty$. In particular, our aim is to exhibit the influence of the number of vanishing moments of the wavelet function on the asymptotic cross-wavelet covariance. Such a result needs the following assumption:

[C3] The wavelet function has $M \geq 1$ vanishing moments that is

$$
\int_{\mathbb{R}} t^{m} \psi(t) \mathrm{d} t=0 \text { for } m=0, \ldots, M-1 \quad \text { and } \quad \int_{\mathbb{R}} t^{M} \psi(t) \mathrm{d} t \neq 0 .
$$

We may now derive our result obtained as $|h| \rightarrow+\infty$. Let us first recall Landau notation: for two functions $f(h)$ and $g(h)$ defined on $\mathbb{R}$, we denote by $f(h) \sim g(h)$ as $|h| \rightarrow+\infty($ resp. $f(h)=o(g(h))$ and $f(h)=\mathcal{O}(g(h)))$ if $\lim _{|h| \rightarrow+\infty} f(h) / g(h)=1$ (resp. $\lim _{|h| \rightarrow+\infty} f(h) / g(h)=0$ and $f(h) / g(h)$ is bounded for all $h$ ).

Theorem 3.2. Assume [C1], [C2(2M+1)] and [C3] hold. Let $a_{1}, a_{2}>0$ and $b, h \in \mathbb{R}$, then as $|h| \rightarrow+\infty$, we have

$$
E\left[d_{a_{1}, b+h}^{j} \overline{d_{a_{2}, b}^{k}}\right] \sim-\frac{\sigma_{j} \sigma_{k}}{2} \kappa\left(\psi, M, a_{1}, a_{2}\right) \tau_{j k}(h)|h|^{H_{j}+H_{k}-2 M}
$$

where $\kappa\left(\psi, M, a_{1}, a_{2}\right):=\left(\begin{array}{c}2 M \\ M\end{array}\right)\left(a_{1} a_{2}\right)^{M}\left|\int t^{M} \psi(t) \mathrm{d} t\right|^{2}$ and

$$
\tau_{j k}(h)= \begin{cases}\left(\rho_{j k}+\eta_{j k} \operatorname{sign}(h)\right)\left(\begin{array}{c}
H_{j}+H_{k} \\
2 M
\end{array}\right) & \text { if } H_{j}+H_{k} \neq 1 \\
-\frac{\eta_{j k} \times \operatorname{sign}(h)}{2 M(2 M-1)} & \text { if } H_{j}+H_{k}=1 .\end{cases}
$$

We notice that the equivalence stated has a meaning as soon as $\rho_{j k}+\eta_{j k} \operatorname{sign}(h) \neq 0$ when $H_{j}+H_{k} \neq 1$ and as soon as $\eta_{j k} \neq 0$ when $H_{j}+H_{k}=1$. In the opposite cases, a careful look at the proof shows that the equivalence can be replaced by an upper-bound or more precisely $\mathbb{E}\left[d_{a_{1}, b+h}^{j} \overline{d_{a_{2}, b}^{k}}\right]=o\left(|h|^{1-2 M}\right)$.

Similarly to the fractional Brownian motion, Theorem 3.2 asserts that the higher $M$, the less correlated the wavelet transforms of the components $j$ and $k$ of the multivariate fractional Brownian motion. This has many implications. In particular, this suggests that estimating the instantaneous cross-wavelet correlation at a scale $a$ may be efficiently done by using the empirical correlation since at scale $a, d_{a, b+h}^{j}$ and $d_{a, b}^{k}$ are not too much correlated if $M$ is large, see Section 4. 


\subsection{Cross-spectral density of the wavelet transform of the $\mathrm{mfBm}$}

In the case of the $\mathrm{fBm}$, the expression of the spectral density of the wavelet transform was provided by Flandrin [12,13]. A rigorous proof of the existence of this spectral density in the $L^{1}$ sense was obtained by Kato and Masry [16]. On the basis of this work, our ambition is to provide the cross-spectral density between wavelet transforms (at different scales) of components $j$ and $k$ of the multivariate fractional Brownian motion. The idea is to obtain the following spectral representation for the cross-correlation

$$
E\left[d_{a_{1}, b+h}^{j} \overline{d_{a_{2}, b}^{k}}\right]=\frac{1}{2 \pi} \int_{\mathbb{R}} S_{a_{1}, a_{2}}^{j k}(\omega) \mathrm{e}^{\mathrm{i} \omega t} \mathrm{~d} \omega .
$$

Theorem 3.3. Under Assumptions [C1], $[\mathrm{C} 2(M)]$ and [C3] (with $M \geq 2$ ), we derive the following assertions for $a_{1}, a_{2}>0$ and $\omega \in \mathbb{R}$ :

(i) The cross-spectral density of the wavelet transforms of two components $j$ and $k$ exists and is given by

$$
S_{a_{1}, a_{2}}^{j k}(\omega)=\sqrt{a_{1} a_{2}} \sigma_{j} \sigma_{k} \Gamma\left(H_{j}+H_{k}+1\right) \zeta_{j k}(\omega) \frac{\overline{\widehat{\psi}\left(a_{1} \omega\right)} \widehat{\psi}\left(a_{2} \omega\right)}{|\omega|^{H_{j}+H_{k}+1}}
$$

where

$$
\zeta_{j k}(\omega)= \begin{cases}\rho_{j k} \sin \left(\frac{\pi}{2}\left(H_{j}+H_{k}\right)\right)+\mathbf{i} \eta_{j k} \cos \left(\frac{\pi}{2}\left(H_{j}+H_{k}\right)\right) \operatorname{sign}(\omega) & \text { if } H_{j}+H_{k} \neq 1 \\ \rho_{j k}+\mathbf{i} \frac{\pi}{2} \eta_{j k} \operatorname{sign}(\omega) & \text { if } H_{j}+H_{k}=1 .\end{cases}
$$

(ii) We have for both cases, as $\omega \rightarrow 0$

$$
\left|S_{a_{1}, a_{2}}^{j k}(\omega)\right| \sim\left(a_{1} a_{2}\right)^{M+1 / 2} \sigma_{j} \sigma_{k} \Gamma\left(H_{j}+H_{k}+1\right)\left|\widehat{\psi}^{(M)}(0)\right|^{2}\left|\zeta_{j k}(\omega)\right||\omega|^{2 M-1-\alpha} .
$$

(iii) Moreover, the coherence function between the two components $j$ and $k$ satisfies:

$$
C_{a_{1}, a_{2}}^{j k}(\omega):=\frac{\left|S_{a_{1}, a_{2}}^{j k}(\omega)\right|^{2}}{S_{a_{1}, a_{1}}^{j j}(\omega) S_{a_{2}, a_{2}}^{k k}(\omega)}=\left|\zeta_{j k}(\omega)\right|^{2} \frac{\Gamma\left(H_{j}+H_{k}+1\right)^{2}}{\Gamma\left(2 H_{j}+1\right) \Gamma\left(2 H_{k}+1\right)} \times \frac{\widehat{\psi}\left(a_{1} \omega\right) \overline{\widehat{\psi}\left(a_{2} \omega\right)}}{\overline{\widehat{\psi}\left(a_{1} \omega\right) \widehat{\psi}\left(a_{2} \omega\right)}} .
$$

Before writing down the proof, let us give some comments.

1. Item (ii) in Theorem 3.3 is the spectral analogue of Theorem 3.2. Indeed the behaviour of the cross-correlation at infinite lags is linked to the behaviour of its Fourier transform at the zero frequency. We recover the fact that as soon as $M>H_{j}+H_{k}+1 / 2$, the long-range interdependence is destroyed. The divergence of $|\omega|^{-1-H_{j}+H_{k}}$ is compensated by the rapid decrease to zero of the Fourier transform of the wavelet.

2. The interpretation of the coherence (10) is difficult here. Indeed, it is complex valued, a property which is not natural for a coherence. This comes from the fact that the quantities $S_{a_{1}, a_{2}}^{j j}(\omega)$ are not power spectral densities but cross-spectral densities (cross-spectral density between two different scales of the wavelet transform of one signal). Thus, to interpret correctly the coherence, we should look at one scale only, in which case we recover the coherence evaluated in the usual spectral domain. And this result is logical since the usual coherence is independent of the frequency.

3. Setting $a_{1}=a_{2}$ and $j=k$ in the expression of the cross-spectral density, we recover the usual result of the power spectral density at one scale of the wavelet transform of a scalar fBm. The proof proposed here is a natural extension of the proof found in Kato and Masry [16].

4. The derivation of the analytic form of the cross-spectral density is easy if we use generalized functions (or Schwartz distributions). Indeed, from Gel'fand and Shilov [14], we know that the Fourier transforms of $|v|^{\alpha}$ and $|v|^{\alpha} \operatorname{sign}(v)$ are respectively given by $-2 \Gamma(\alpha+1)|\omega|^{-\alpha-1} \sin (\pi \alpha / 2)$ and $-2 \mathbf{i} \Gamma(\alpha+1)|\omega|^{-\alpha-1} \operatorname{sign}(\omega) \sin (\pi \alpha / 2)$. Then, in the proof below, the calculation of $T=\int_{\mathbb{R}} w_{j k}(v) \overline{\Gamma_{\psi}}(v) \mathrm{d} v$ can be done using Parseval equality. However, the theoretical background required and hidden in the calculation is far more involved than the basics we have used in the proof (see [14]). 


\section{Discussion}

To estimate the Hurst exponent of a monovariate fractional Brownian motion using wavelet techniques or filtering techniques, the key-ingredient is that the logarithm of the wavelet variance is linear in the logarithm of the scale which suggests to estimate the Hurst exponent via the slope from the regression of the log-scalogram on the logarithm of the scales for a set of different scales as investigated for instance by Veitch and Abry [21], Bardet et al. [7], Faÿ et al. [11]. Since each component of a $\mathrm{mfBm}$ is a $\mathrm{fBm}$, this procedure can still be efficiently considered to estimate the different Hurst exponents. Proposition 3.1 provides an additional information. Indeed, this result asserts in particular that the logarithm of the absolute cross-wavelet variance between two components $j$ and $k$ also exhibits a linear behaviour with the logarithm of the scale with proportionality coefficient equal to $H_{j}+H_{k}$. This theoretical fact clearly suggests to include the information of the logarithm of the asbsolute empirical cross-wavelet variance in order to improve the classical procedure. Such ideas have been considered in details in Amblard and Coeurjolly [3], where we propose a joint estimation of the parameters $H_{j}$ for $j=1, \ldots, p$ and also efficient procedures to estimate $\sigma_{j}^{2}, \rho_{j k}$ and $\eta_{j k}$ for $j, k=1, \ldots, p, k>j$. In particular for specific wavelet, we exploit Theorem 3.2 to obtain a central limit theorem for the sample variances at different scales of the wavelet transforms as well as the sample cross-correlations. From this, we derived a multivariate central limit theorem for the estimators.

\section{Appendix A. Proofs of the main Results}

\section{A.1. Proof of Theorem 3.2}

Proof. The proof is split into two cases. Before this, we denote for $h>0$ by $D_{h}:=\left\{\left(t_{1}, t_{2}\right)\right.$ $\left.\in \mathbb{R}^{2}:\left|a_{2} t_{2}-a_{1} t_{1}\right|<\frac{|h|}{2}\right\}$ and we note in particular that

$$
\begin{aligned}
& \forall\left(t_{1}, t_{2}\right) \in D_{h}\left|\frac{a_{2} t_{2}-a_{1} t_{1}}{h}\right| \leq \frac{1}{2}<1 \text { and } \operatorname{sign}\left(a_{2} t_{2}-a_{1} t_{1}-h\right)=-\operatorname{sign}(h) \\
& \forall\left(t_{1}, t_{2}\right) \in \mathbb{R}^{2} \backslash D_{h}\left|\frac{h}{a_{2} t_{2}-a_{1} t_{1}}\right| \leq 2 .
\end{aligned}
$$

For brevity, we assume in the following that for for $t_{1}, t_{2} \in \mathbb{R}, a_{1} t_{1} \neq a_{2} t_{2}$.

Case 1. $\alpha:=H_{j}+H_{k} \neq 1$.

We assume here that $\rho_{j k}+\eta_{j k} \operatorname{sign}(h) \neq 0$. Let us write $E\left[d_{a, b+h}^{j} \overline{d_{a, b}^{k}}\right]=-\frac{\sigma_{j} \sigma_{k}}{2} \sqrt{a_{1} a_{2}} \times T$ with

$$
T:=\int_{\mathbb{R}^{2}}\left(\rho_{j k}-\eta_{j k} \operatorname{sign}\left(a_{2} t_{2}-a_{1} t_{1}-h\right)\right)\left|a_{2} t_{2}-a_{1} t_{1}-h\right|^{\alpha} \bar{\psi}\left(t_{1}\right) \psi\left(t_{2}\right) \mathrm{d} t_{1} \mathrm{~d} t_{2}=T_{1}+T_{2},
$$

and where $T_{1}$ (resp. $T_{2}$ ) corresponds to the integral on $D_{h}$ (resp. $\mathbb{R}^{2} \backslash D_{h}$ ). Let us first prove that $|h|^{2 M-\alpha} T_{2} \rightarrow 0$ as $|h| \rightarrow+\infty$. Denoting $c^{\vee}=\left|\rho_{j k}\right|+\left|\eta_{j k}\right|$, we have since $2 M-\alpha>0$ and from (A.2) for the second inequality

$$
\begin{aligned}
|h|^{2 M-\alpha}\left|T_{2}\right| & \leq c^{\vee} \int_{\mathbb{R}^{2} \backslash D_{h}}\left|a_{2} t_{2}-a_{1} t_{1}\right|^{\alpha}|h|^{2 M-\alpha}\left|1-\frac{h}{a_{2} t_{2}-a_{1} t_{1}}\right|^{\alpha}\left|\psi\left(t_{1}\right)\right|\left|\psi\left(t_{2}\right)\right| \mathrm{d} t_{1} \mathrm{~d} t_{2} \\
& \leq 2^{2 M-\alpha} 3^{\alpha} c^{\vee} \int_{\mathbb{R}^{2} \backslash D_{h}}\left(a_{2} t_{2}-a_{1} t_{1}\right)^{2 M}\left|\psi\left(t_{1}\right)\right|\left|\psi\left(t_{2}\right)\right| \mathrm{d} t_{1} \mathrm{~d} t_{2} .
\end{aligned}
$$


The result is then obtained by using assumption $[\mathrm{C} 2(2 M)]$ and the dominated convergence theorem. Now, within the domain $D_{h}$, one may use the series expansion of $(1+x)^{\alpha}$ (for $\left.|x|<1\right)$.

$$
\begin{aligned}
T_{1} & =|h|^{\alpha} \int_{D_{h}}\left(\rho_{j k}-\eta_{j k} \operatorname{sign}\left(a_{2} t_{2}-a_{1} t_{1}-h\right)\right)\left(1-\frac{a_{2} t_{2}-a_{1} t_{1}}{h}\right)^{\alpha} \bar{\psi}\left(t_{1}\right) \psi\left(t_{2}\right) \mathrm{d} t_{1} \mathrm{~d} t_{2} \\
& =|h|^{\alpha}\left(\rho_{j k}+\eta_{j k} \operatorname{sign}(h)\right) \int_{D_{h}}\left(\sum_{\ell \geq 0}(-1)^{\ell}\left(\begin{array}{c}
\alpha \\
\ell
\end{array}\right)\left(\frac{a_{2} t_{2}-a_{1} t_{1}}{h}\right)^{\ell}\right) \bar{\psi}\left(t_{1}\right) \psi\left(t_{2}\right) \mathrm{d} t_{1} \mathrm{~d} t_{2},
\end{aligned}
$$

where $\left(\begin{array}{c}\alpha \\ \ell\end{array}\right)$ denotes the binomial coefficient $(\alpha)(\alpha-1) \ldots(\alpha-\ell+1) / \ell$ !. Decompose $T_{1}$ into three terms (denoted by $T_{1}^{\prime}, T_{2}^{\prime}$ and $\left.T_{3}^{\prime}\right)$ corresponding to the $2 M$ first terms of the series, the $(2 M+1)$ th term $(\ell=2 M)$ and the remainder terms. Then,

$$
T_{1}^{\prime}=|h|^{\alpha}\left(\rho_{j k}+\eta_{j k} \operatorname{sign}(h)\right) \sum_{\ell=0}^{2 M-1}(-1)^{\ell} h^{-\ell}\left(\begin{array}{l}
\alpha \\
\ell
\end{array}\right) \int_{D_{h}}\left(a_{2} t_{2}-a_{1} t_{1}\right)^{\ell} \bar{\psi}\left(t_{1}\right) \psi\left(t_{2}\right) \mathrm{d} t_{1} \mathrm{~d} t_{2} .
$$

Under Assumption [C3], $\psi$ has $M$ vanishing moments and therefore the previous expression reduces to

$$
T_{1}^{\prime}=-|h|^{\alpha}\left(\rho_{j k}+\eta_{j k} \operatorname{sign}(h)\right) \sum_{\ell=0}^{2 M-1}(-1)^{\ell} h^{-\ell}\left(\begin{array}{c}
\alpha \\
\ell
\end{array}\right) \int_{\mathbb{R}^{2} \backslash D_{h}}\left(a_{2} t_{2}-a_{1} t_{1}\right)^{\ell} \bar{\psi}\left(t_{1}\right) \psi\left(t_{2}\right) \mathrm{d} t_{1} \mathrm{~d} t_{2} .
$$

Now,

$$
|h|^{2 M-\alpha}\left|T_{1}^{\prime}\right| \leq c^{\vee} \sum_{\ell=0}^{2 M-1}\left|\left(\begin{array}{l}
\alpha \\
\ell
\end{array}\right)\right| \int_{\mathbb{R}^{2} \backslash D_{h}} 2^{2 M-\ell}\left(a_{2} t_{2}-a_{1} t_{1}\right)^{2 M}\left|\psi\left(t_{1}\right)\right|\left|\psi\left(t_{2}\right)\right| \mathrm{d} t_{1} \mathrm{~d} t_{2} .
$$

Since $\mathbb{R}^{2} \backslash D_{h} \rightarrow 0$ as $|h| \rightarrow+\infty$, Assumption $[\mathrm{C} 2(2 M)]$ and the dominated convergence theorem may be combined to prove that $|h|^{2 M-\alpha} T_{1}^{\prime} \rightarrow 0$ as $|h| \rightarrow+\infty$. The term $T_{2}^{\prime}$ is defined as

$$
T_{2}^{\prime}:=|h|^{\alpha-2 M}\left(\rho_{j k}+\eta_{j k} \operatorname{sign}(h)\right)\left(\begin{array}{c}
\alpha \\
2 M
\end{array}\right) \int_{D_{h}}\left(a_{2} t_{2}-a_{1} t_{1}\right)^{2 M} \bar{\psi}\left(t_{1}\right) \psi\left(t_{2}\right) \mathrm{d} t_{1} \mathrm{~d} t_{2} .
$$

As previously we obtain

$$
\begin{aligned}
\frac{|h|^{2 M-\alpha} T_{2}^{\prime}}{\rho_{j k}+\eta_{j k} \operatorname{sign}(h)} & \rightarrow\left(\begin{array}{c}
\alpha \\
2 M
\end{array}\right) \int_{\mathbb{R}^{2}}\left(a_{2} t_{2}-a_{1} t_{1}\right)^{2 M} \bar{\psi}\left(t_{1}\right) \psi\left(t_{2}\right) \mathrm{d} t_{1} \mathrm{~d} t_{2} \\
& =\left(\begin{array}{c}
\alpha \\
2 M
\end{array}\right)\left(\begin{array}{c}
2 M \\
M
\end{array}\right)\left(a_{1} a_{2}\right)^{M}\left|\int t^{M} \psi(t) \mathrm{d} t\right|^{2}=\left(\begin{array}{c}
\alpha \\
2 M
\end{array}\right) \kappa\left(\psi, M, a_{1}, a_{2}\right) .
\end{aligned}
$$

Since $T=T_{1}+T_{2}=T_{1}^{\prime}+T_{2}^{\prime}+T_{3}^{\prime}+T_{2}$, the proof will be completed if we manage to prove that $|h|^{2 M-\alpha} T_{3}^{\prime} \rightarrow 0$. Let us write

$$
\begin{aligned}
|h|^{2 M-\alpha} T_{3}^{\prime}= & h^{2 M}\left(\rho_{j k}+\eta_{j k} \operatorname{sign}(h)\right) \int_{D_{h}} \sum_{\ell \geq 2 M+1}(-1)^{\ell}\left(\begin{array}{c}
\alpha \\
\ell
\end{array}\right)\left(\frac{a_{2} t_{2}-a_{1} t_{1}}{h}\right)^{\ell} \bar{\psi}\left(t_{1}\right) \psi\left(t_{2}\right) \mathrm{d} t_{1} \mathrm{~d} t_{2} \\
= & \frac{\rho_{j k}+\eta_{j k} \operatorname{sign}(h)}{h} \\
& \times \int_{D_{h}}\left(a_{2} t_{2}-a_{1} t_{1}\right)^{2 M+1}\left(\sum_{\ell \geq 0}(-1)^{\ell+1}\left(\begin{array}{c}
\alpha \\
\ell+2 M+1
\end{array}\right)\left(\frac{a_{2} t_{2}-a_{1} t_{1}}{h}\right)^{\ell}\right) \bar{\psi}\left(t_{1}\right) \psi\left(t_{2}\right) \mathrm{d} t_{1} \mathrm{~d} t_{2} .
\end{aligned}
$$


The binomial coefficient appearing in the last equation satisfies, with $\ell^{\prime}=\ell+2 M+1$

$$
\begin{aligned}
\left|\left(\begin{array}{l}
\alpha \\
\ell^{\prime}
\end{array}\right)\right| & =\frac{\left|\alpha(\alpha-1) \cdots\left(\alpha-\ell^{\prime}+1\right)\right|}{\ell^{\prime} !} \\
& \leq \frac{2(2-\alpha) \cdots\left(\ell^{\prime}-1-\alpha\right)}{\ell^{\prime} !} \quad \text { since } \alpha \leq 2 \\
& \leq \frac{2\left(\ell^{\prime}-1\right) !}{\ell^{\prime} !}=\frac{2}{\ell^{\prime}} \leq \frac{2}{\ell} .
\end{aligned}
$$

Recall that in $D_{h}$ we have $\left|a_{2} t_{2}-a_{1} t_{1}\right| /|h| \leq 1 / 2$. From (A.1), the series in the previous integral then satisfies

$$
\begin{aligned}
\left|\sum_{\ell \geq 0}\left(\begin{array}{c}
\alpha \\
\ell+2 M+1
\end{array}\right)\left(\frac{a_{2} t_{2}-a_{1} t_{1}}{h}\right)^{\ell}\right| & \leq\left|\left(\begin{array}{c}
\alpha \\
2 M+1
\end{array}\right)\right|+\sum_{\ell \geq 1}\left|\left(\begin{array}{c}
\alpha \\
\ell+2 M+1
\end{array}\right)\right|\left|\frac{a_{2} t_{2}-a_{1} t_{1}}{h}\right|^{\ell} \\
& \leq \frac{2}{2 M+1}+\sum_{\ell \geq 1} \frac{2}{\ell} 2^{-\ell} \\
& =\frac{2}{2 M+1}+2 \log (2)=: C_{M} .
\end{aligned}
$$

Thus we obtain

$$
|h|^{2 M-\alpha}\left|T_{3}^{\prime}\right| \leq \frac{C_{M} c^{\vee}}{|h|} \int_{\mathbb{R}^{2}}\left|a_{2} t_{2}-a_{1} t_{1}\right|^{2 M+1}\left|\psi\left(t_{1}\right)\right|\left|\psi\left(t_{2}\right)\right| \mathrm{d} t_{1} \mathrm{~d} t_{2}
$$

Since by Assumption $[\mathrm{C} 2(2 M+1)], t^{2 M+1} \psi(t) \in L^{1}$, we have $|h|^{2 M-\alpha}\left|T_{3}^{\prime}\right|=O\left(|h|^{-1}\right)$, whence the result.

Case 2. $H_{j}+H_{k}=1$.

We assume here that $\eta_{j k} \neq 0$. We take the same notation as previously. We first note that, under [C1], the term $T$ can be rewritten as

$$
T=\int_{\mathbb{R}^{2}} \rho_{j k}\left|a_{2} t_{2}-a_{1} t_{1}-h\right|+\eta_{j k}\left(a_{2} t_{2}-a_{1} t_{1}-h\right) \log \left|1-\frac{a_{2} t_{2}-a_{1} t_{1}}{h}\right| \bar{\psi}\left(t_{1}\right) \psi\left(t_{2}\right) \mathrm{d} t_{1} \mathrm{~d} t_{2} .
$$

We decompose $T$ in $T_{1}+T_{2}$ (as done in case 1 ). The proof that $|h|^{2 M-1} T_{2} \rightarrow 0$ as $|h| \rightarrow+\infty$ follows similar arguments as in the case 1 and is therefore omitted. Now, the term $T_{1}$ can be rewritten as

$$
\begin{aligned}
T_{1}= & \rho_{j k}|h| \int_{D_{h}}\left(1-\frac{a_{2} t_{2}-a_{1} t_{1}}{h}\right) \bar{\psi}\left(t_{1}\right) \psi\left(t_{2}\right) \mathrm{d} t_{1} \mathrm{~d} t_{2} \\
& -\eta_{j k} h \int_{D_{h}}\left(1-\frac{a_{2} t_{2}-a_{1} t_{1}}{h}\right) \log \left(1-\frac{a_{2} t_{2}-a_{1} t_{1}}{h}\right) \bar{\psi}\left(t_{1}\right) \psi\left(t_{2}\right) \mathrm{d} t_{1} \mathrm{~d} t_{2} .
\end{aligned}
$$

Denote by $\tilde{T}_{1}$ and $\tilde{T}_{2}$ these two terms. Assumption [C1] leads to

$$
\begin{aligned}
\tilde{T}_{1} & =-\rho_{j k}|h| \int_{\mathbb{R}^{2} \backslash D_{h}}\left(1-\frac{a_{2} t_{2}-a_{1} t_{1}}{h}\right) \bar{\psi}\left(t_{1}\right) \psi\left(t_{2}\right) \mathrm{d} t_{1} \mathrm{~d} t_{2} \\
& =-\rho_{j k} \int_{\mathbb{R}^{2} \backslash D_{h}}\left|a_{2} t_{2}-a_{1} t_{1}\right|\left|1-\frac{h}{a_{2} t_{2}-a_{1} t_{1}}\right| \mathrm{d} t_{1} \mathrm{~d} t_{2} .
\end{aligned}
$$

Then, we assert that

$$
|h|^{2 M-1}\left|\tilde{T}_{1}\right| \leq 2^{2 M-1} 3\left|\rho_{j k}\right| \int_{\mathbb{R}^{2} \backslash D_{h}}\left(a_{2} t_{2}-a_{1} t_{1}\right)^{2 M}\left|\psi\left(t_{1}\right)\right|\left|\psi\left(t_{2}\right)\right| \mathrm{d} t_{1} \mathrm{~d} t_{2} \rightarrow 0
$$


as $|h| \rightarrow+\infty$. For the term $\tilde{T}_{2}$, we may use the series expansion of $\log (1+x)$ (for $\left.|x|<1\right)$. We omit the details and leave the reader to verify that as $|h| \rightarrow+\infty$

$$
\begin{aligned}
\tilde{T}_{2} \sim & \eta_{j k} h \int_{\mathbb{R}^{2}}\left(1-\frac{a_{2} t_{2}-a_{1} t_{1}}{h}\right)\left(\frac{-1}{2 M-1}\left(\frac{a_{2} t_{2}-a_{1} t_{1}}{h}\right)^{2 M-1}\right. \\
& \left.\times \frac{-1}{2 M}\left(\frac{a_{2} t_{2}-a_{1} t_{1}}{h}\right)^{2 M}\right) \bar{\psi}\left(t_{1}\right) \psi\left(t_{2}\right) \mathrm{d} t_{1} \mathrm{~d} t_{2} \\
& \sim-h^{1-2 M} \times \frac{\eta_{j k}}{2 M(2 M-1)}\left(\begin{array}{c}
2 M \\
M
\end{array}\right)\left(a_{1} a_{2}\right)^{M}\left|\int t^{M} \psi(t) \mathrm{d} t\right|^{2} .
\end{aligned}
$$

Hence, $T \sim|h|^{1-2 M} \times\left(-\frac{\eta_{j k} \times \operatorname{sign}(h)}{2 M(2 M-1)}\right) \kappa\left(\psi, M, a_{1}, a_{2}\right)$.

In this proof, Fubini's theorem and interchanges of integrals and (in)finite sums are widely used. All of these are justified by the absolute convergence of the different series related to the expansions of $(1+x)^{\alpha}$ or $\log (1+x)$ for $|x|<1$ and Assumption [C2(2M+1)].

\section{A.2. Proof of Theorem 3.3}

Proof. (i) We recall that under [C1] and [C2(2)], equation (5) holds, that is $E\left[d_{a_{1}, b_{1}}^{j} \overline{d_{a_{2}, b_{2}}^{k}}\right]=-\frac{\sigma_{j} \sigma_{k}}{2} T$ with $T:=\int_{\mathbb{R}} w_{j k}(v) \overline{\Gamma_{\psi}}(v) \mathrm{d} v$. Furthermore, note that the Fourier transforms of $\psi_{a, b}$ and $\overline{\Gamma_{\psi}}(v)$ exist and are equal respectively to $\sqrt{a} \widehat{\psi}(a \omega) \mathrm{e}^{-\mathbf{i} \omega b}$ and to

$$
q(\omega):=\widehat{\overline{\Gamma_{\psi}}}(\omega)=\int_{\mathbb{R}} \overline{\Gamma_{\psi}}(v) \mathrm{e}^{-\mathrm{i} \omega v} \mathrm{~d} v=\sqrt{a_{1} a_{2}} \overline{\widehat{\psi}\left(a_{1} \omega\right)} \widehat{\psi}\left(a_{2} \omega\right) \mathrm{e}^{\mathrm{i} \omega h} .
$$

Now, let us split the proof into two cases.

Case 1. $\alpha:=H_{j}+H_{k} \neq 1$.

When $j=k$, at this step, Kato and Masry [16] have used the representation of $|v|^{\alpha}$ obtained by von Bahr and Esseen [22]. We have obtained a similar representation for the function $\operatorname{sign}(v)|v|^{\alpha}$ for $\alpha \in(0,2) \backslash\{1\}$ (see Eqs. (A.5) and (A.6) in Lem. A.1). Let $g_{\alpha}: \mathbb{R} \rightarrow \mathbb{R}$ be the function which equals zero when $\alpha \in(0,1)$ and which is the identity function when $\alpha \in(1,2)$. We have by Fubini's theorem and under Assumption [C3] (with $M \geq 2)$.

$$
\begin{aligned}
T= & \int_{\mathbb{R}}\left(\rho_{j k}-\eta_{j k} \operatorname{sign}(v)\right)|v|^{\alpha} \overline{\Gamma_{\psi}}(v) \mathrm{d} v \\
= & \frac{\Gamma(\alpha+1)}{\pi} \int_{\mathbb{R}}|\omega|^{-\alpha-1} \int_{\mathbb{R}}\left(\rho_{j k} \sin (\pi \alpha / 2)(1-\cos (\omega v))-\eta_{j k} \cos (\pi \alpha / 2) \operatorname{sign}(\omega)\left(\sin (\omega v)-g_{\alpha}(\omega v)\right)\right) \\
& \times \overline{\Gamma_{\psi}}(v) \mathrm{d} v \mathrm{~d} \omega \\
= & \frac{\Gamma(\alpha+1)}{\pi} \int_{\mathbb{R}}|\omega|^{-\alpha-1}\left(-\rho_{j k} \sin (\pi \alpha / 2)\left(\frac{q(-\omega)+q(\omega)}{2}\right)-\eta_{j k} \cos (\pi \alpha / 2) \operatorname{sign}(\omega)\left(\frac{q(-\omega)-q(\omega)}{2 \mathbf{i}}\right)\right) \mathrm{d} \omega \\
= & -\frac{\Gamma(\alpha+1)}{\pi} \int_{\mathbb{R}}|\omega|^{-\alpha-1}\left(\rho_{j k} \sin (\pi \alpha / 2)+\mathbf{i} \eta_{j k} \cos (\pi \alpha / 2) \operatorname{sign}(\omega)\right) q(\omega) \mathrm{d} \omega \\
= & -\frac{\Gamma(\alpha+1)}{\pi} \int_{\mathbb{R}}|\omega|^{-\alpha-1} \zeta_{j k}(\omega) q(\omega) \mathrm{d} \omega .
\end{aligned}
$$

Note that the condition $M \geq 2$ is required for $\alpha>1$. For $\alpha<1, M \geq 1$ is a sufficient condition. These conditions allow us to show that the contributions $\int \overline{\Gamma_{\psi}}(v) \mathrm{d} v$ and $\int_{\mathbb{R}} g_{\alpha}(\omega v) \overline{\Gamma_{\psi}}(v) \mathrm{d} v$ are equal to zero. Now, using (A.3) 
we obtain

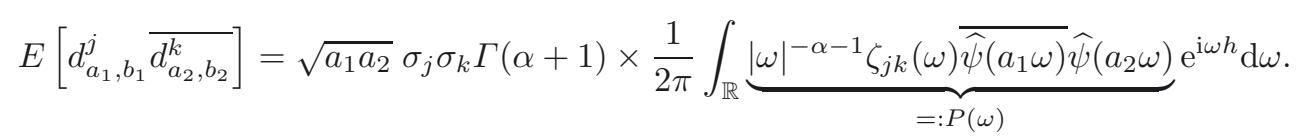

By using Bochner's theorem, the proof will be done, if one proves that the function $P(\cdot)$ is integrable. Let us prove this last assertion. Under $[\mathrm{C} 2(\mathrm{M})], t^{k} \psi(t) \in L^{1}$ for $k=0, \ldots, M$. Therefore, $\widehat{\psi}$ is a $M$ times continuous and differentiable function. Using a Taylor expansion

$$
\widehat{\psi}(\omega)=\sum_{k=0}^{M-1} \omega^{k} \widehat{\psi}^{(k)}(\omega)+\omega^{M} \widehat{\psi}^{(M)}(\widetilde{\omega})=\omega^{M} \widehat{\psi}^{(M)}(\widetilde{\omega}), \text { with } \widetilde{\omega} \in[0 \wedge \omega, 0 \vee \omega],
$$

under $[\mathrm{C} 2(\mathrm{M})]$. And since $\psi^{(M)}$ is continuous at zero, $\widehat{\psi}(\omega) \sim \omega^{M} \widehat{\psi}^{(M)}(0)$ as $\omega \rightarrow 0$. Then as $\omega \rightarrow 0$ :

$$
P(\omega) \sim \zeta_{j k}(\omega)|\omega|^{2 M-1-\alpha}\left(a_{1} a_{2}\right)^{M}\left|\widehat{\psi}^{(M)}(0)\right|^{2} .
$$

As a consequence, for $M \geq 2, P$ is continuous at zero and $\lim _{\omega \rightarrow 0^{ \pm}} P(\omega)=0$. Therefore for $\varepsilon>0, P$ is integrable on the interval $[-\varepsilon, \varepsilon]$ as a continuous function on this interval. Finally (with $c^{\vee}:=\left|\rho_{j k}\right|+\left|\eta_{k j}\right|$ ),

$$
\begin{aligned}
\int_{|\omega| \geq \varepsilon}|P(\omega)| & \leq c^{\vee}\left(a_{1}^{\alpha} \int_{|\omega| \geq a_{1} \varepsilon} \frac{|\widehat{\psi}(\omega)|^{2}}{|\omega|^{\alpha+1}} \mathrm{~d} \omega\right)^{1 / 2}\left(a_{2}^{\alpha} \int_{|\omega| \geq a_{2} \varepsilon} \frac{|\widehat{\psi}(\omega)|^{2}}{|\omega|^{\alpha+1}} \mathrm{~d} \omega\right)^{1 / 2} \\
& \leq \frac{c^{\vee}}{\varepsilon^{\alpha}} \int_{\mathbb{R}} \frac{|\widehat{\psi}(\omega)|^{2}}{|\omega|} \mathrm{d} \omega<+\infty,
\end{aligned}
$$

under $[\mathrm{C} 1]$. Hence, $P(\cdot) \in L^{1}$ and Bochner's theorem may be applied.

Case 2. $H_{j}+H_{k}=1$.

We start with the representation of $v \log |v|$ given by (A.8).

$$
\begin{aligned}
w_{j k}(v) & =\rho_{j k}|v|+\eta_{j k} v \log |v|=\lim _{\alpha \rightarrow 1^{-}} \rho_{j k}|v|^{\alpha}+\eta_{j k} v \log |v| \\
& =\lim _{\alpha \rightarrow 1^{-}} \frac{1}{2 \pi} \int_{\mathbb{R}} \frac{2 \rho_{j k}(1-\cos (\omega v))-\pi \eta_{j k} \operatorname{sign}(\omega) \sin (\omega v)}{|\omega|^{\alpha+1}} \mathrm{~d} \omega .
\end{aligned}
$$

Now, we derive the computation of the term $T:=\int_{\mathbb{R}} w_{j k}(v) \bar{\Gamma}_{\psi}(-v) \mathrm{d} v$, similarly as the previous case. Using dominated convergence theorem and Fubini's theorem,

$$
\begin{aligned}
T & =\frac{1}{2 \pi} \int_{\mathbb{R}}\left(\lim _{\alpha \rightarrow 1^{-}} \int_{\mathbb{R}} \frac{2 \rho_{j k}(1-\cos (\omega v))-\pi \eta_{j k} \operatorname{sign}(\omega) \sin (\omega v)}{|\omega|^{\alpha+1}} \mathrm{~d} \omega\right) \bar{\Gamma}_{\psi}(v) \mathrm{d} v \\
& =\frac{1}{2 \pi} \lim _{\alpha \rightarrow 1^{-}} \int_{\mathbb{R}}\left(\int_{\mathbb{R}} \frac{2 \rho_{j k}(1-\cos (\omega v))-\pi \eta_{j k} \operatorname{sign}(\omega) \sin (\omega v)}{|\omega|^{\alpha+1}} \bar{\Gamma}_{\psi}(v) \mathrm{d} v\right) \mathrm{d} \omega \\
& =\frac{1}{2 \pi} \lim _{\alpha \rightarrow 1^{-}} \int_{\mathbb{R}}\left(-2 \rho_{j k}\left(\frac{q(-\omega)+q(\omega)}{2}\right)-\pi \eta_{j k} \operatorname{sign}(\omega)\left(\frac{q(-\omega)-q(\omega)}{2 \mathbf{i}}\right)\right)|\omega|^{-\alpha-1} \mathrm{~d} \omega \\
& =-\frac{1}{2 \pi} \lim _{\alpha \rightarrow 1^{-}} \int_{\mathbb{R}} \frac{2 \rho_{j k}+\mathbf{i} \pi \eta_{j k} \operatorname{sign}(\omega)}{|\omega|^{\alpha+1}} q(\omega) \mathrm{d} \omega \\
& =-\frac{1}{2 \pi} \lim _{\alpha \rightarrow 1^{-}} \int_{\mathbb{R}}|\omega|^{-\alpha-1}\left(2 \rho_{j k}+\mathbf{i} \pi \eta_{j k} \operatorname{sign}(\omega)\right) \overline{\hat{\psi}\left(a_{1} \omega\right)} \widehat{\psi}\left(a_{2} \omega\right) \mathrm{e}^{\mathrm{i} \omega h} \mathrm{~d} \omega .
\end{aligned}
$$


From (A.4), $|\omega|^{-\alpha-1} \overline{\widehat{\psi}\left(a_{1} \omega\right)} \widehat{\psi}\left(a_{2} \omega\right)$ is an integrable function for all $\alpha \in(0,2)$. Therefore, the integral and the limit may be interchanged. Therefore, we obtain

$$
E\left[d_{a_{1}, b+h}^{j} \overline{d_{a_{2}, b}^{k}}\right]=\sqrt{a_{1} a_{2}} \sigma_{j} \sigma_{k} \times \frac{1}{2 \pi} \int_{\mathbb{R}} \frac{\rho_{j k}+\mathbf{i} \frac{\pi}{2} \eta_{j k} \operatorname{sign}(\omega)}{|\omega|^{2}} \overline{\widehat{\psi}\left(a_{1} \omega\right)} \widehat{\psi}\left(a_{2} \omega\right) \mathrm{e}^{\mathrm{i} \omega h} \mathrm{~d} \omega,
$$

and Bochner's theorem can be applied.

(ii) is derived from (A.4).

\section{A.3. Bahr and Essen type representations for the functions $\operatorname{sign}(v)|v|^{\alpha}, v_{+}^{\alpha}$ and $v_{-}^{\alpha}$}

In 1965, von Bahr and Essen have obtained the following representation theorem for $|v|^{\alpha}$ for $\alpha \in(0,2)$ :

$$
|v|^{\alpha}=\frac{\Gamma(\alpha+1) \sin (\pi \alpha / 2)}{\pi} \int_{\mathbb{R}} \frac{1-\cos (\omega v)}{|\omega|^{\alpha+1}} \mathrm{~d} \omega .
$$

The following lemma provides a similar representation for $\operatorname{sign}(v)|v|^{\alpha}, v_{+}^{\alpha}=v^{\alpha} \mathbf{1}_{\mathbb{R}^{+}}(v)$ and $v_{-}^{\alpha}=(-v)^{\alpha} \mathbf{1}_{\mathbb{R}^{-}}(v)$.

Lemma A.1. Let $\alpha \in(0,2) \backslash\{1\}$ and let $g_{\alpha}: \mathbb{R} \rightarrow \mathbb{R}$ the function which equals zero when $\alpha \in(0,1)$ and which is the identity function when $\alpha \in(1,2)$, then we have

$$
\begin{aligned}
\operatorname{sign}(v)|v|^{\alpha} & =\frac{\Gamma(\alpha+1) \cos (\pi \alpha / 2)}{\pi} \int_{\mathbb{R}} \frac{\operatorname{sign}(\omega)\left(\sin (\omega v)-g_{\alpha}(\omega v)\right)}{|\omega|^{\alpha+1}} \mathrm{~d} \omega, \\
v_{+}^{\alpha} & =\frac{\Gamma(\alpha+1)}{2 \pi} \int_{\mathbb{R}} \frac{\sin \left(\pi \frac{\alpha}{2}\right)(1-\cos (\omega v))+\cos \left(\pi \frac{\alpha}{2}\right) \operatorname{sign}(\omega)\left(\sin (\omega v)-g_{\alpha}(\omega v)\right)}{|\omega|^{\alpha+1}} \mathrm{~d} \omega, \\
v_{-}^{\alpha} & =\frac{\Gamma(\alpha+1)}{2 \pi} \int_{\mathbb{R}} \frac{\sin \left(\pi \frac{\alpha}{2}\right)(1-\cos (\omega v))-\cos \left(\pi \frac{\alpha}{2}\right) \operatorname{sign}(\omega)(\sin (\omega v)-g(\omega v))}{|\omega|^{\alpha+1}} \mathrm{~d} \omega .
\end{aligned}
$$

The representations of $v_{+}^{\alpha}$ and $v_{-}^{\alpha}$ are obtained from (A.5) and (A.6) noticing that

$$
v_{+}^{\alpha}=\frac{1}{2}\left(|v|^{\alpha}+\operatorname{sign}(v)|v|^{\alpha}\right) \text { and } v_{-}^{\alpha}=\frac{1}{2}\left(|v|^{\alpha}-\operatorname{sign}(v)|v|^{\alpha}\right) .
$$

Proof. Let $\alpha \in(0,1)$, then from (A.5) and properties of the function $\Gamma$

$$
\frac{1}{\alpha+1}|v|^{\alpha+1}=\frac{\Gamma(\alpha+1)}{\pi} \cos (\pi \alpha / 2) \int_{\mathbb{R}} \frac{1-\cos (\omega v)}{|\omega|^{\alpha+2}} \mathrm{~d} \omega .
$$

Since $\int_{\mathbb{R}}|\omega|^{-\alpha-1}|\sin (\omega v)|<+\infty$ for $\alpha \in(0,1)$, we can differentiate this integral with respect to $v$ to obtain

$$
\operatorname{sign}(v)|v|^{\alpha}=\frac{\Gamma(\alpha+1)}{\pi} \cos (\pi \alpha / 2) \int_{\mathbb{R}} \frac{\operatorname{sign}(\omega) \sin (\omega v)}{|\omega|^{\alpha+1}} \mathrm{~d} \omega .
$$

When $\alpha \in(1,2)$, then from (A.5) and properties of the function $\Gamma$

$$
\alpha|v|^{\alpha-1}=\frac{\Gamma(\alpha+1)}{\pi}(-\cos (\pi \alpha / 2)) \int_{\mathbb{R}} \frac{1-\cos (\omega v)}{|\omega|^{\alpha}} \mathrm{d} \omega .
$$

Since $\int_{\mathbb{R}}|\omega|^{-\alpha-1}|\sin (\omega v)-\omega v| \mathrm{d} \omega<+\infty$ for $\alpha \in(1,2)$, we can take the primitive of the last equation to get

$$
\begin{aligned}
\operatorname{sign}(v)|v|^{\alpha} & =\frac{\Gamma(\alpha+1)}{\pi} \cos (\pi \alpha / 2) \int_{\mathbb{R}} \frac{\sin (\omega v) / \omega-v}{|\omega|^{\alpha}} \mathrm{d} \omega \\
& =\frac{\Gamma(\alpha+1)}{\pi} \cos (\pi \alpha / 2) \int_{\mathbb{R}} \frac{\operatorname{sign}(\omega)(\sin (\omega v)-\omega v)}{|\omega|^{\alpha+1}} \mathrm{~d} \omega,
\end{aligned}
$$

which ends the proof. 
Let $\alpha \in(0,1)$, then by differentiating (A.7) with respect to $\alpha$ and taking the limit as $\alpha \rightarrow 1^{-}$, we may obtain

$$
\operatorname{sign}(v)|v| \log |v|=v \log |v|=\lim _{\alpha \rightarrow 1^{-}}-\frac{1}{2} \int_{\mathbb{R}} \frac{\operatorname{sign}(\omega) \sin (\omega v)}{|\omega|^{\alpha+1}} \mathrm{~d} \omega .
$$

Acknowledgements. The authors are sincerely grateful to both referees for their careful reading and their suggestions. This research was supported by the ANR STARAC grant, a fellowship from Region Rhone-Alpes (France), a Marie-Curie International Outgoing Fellowship from the European Community and by ANR InfoNetComaBrain. This research was done while the first author was visiting the Gipsa-lab. He would like to thank the members of the Signal and Images Department for their kind hospitality.

\section{REFERENCES}

[1] S. Achard, R. Salvador, B. Whitcher, J. Suckling and E. Bullmore, A resilient, low-frequency, small-world human brain functional network with highly connected association cortical hubs. J. Neurosci. 26 (2006) 63-72.

[2] S. Achard, D.S. Bassett, A. Meyer-Lindenberg and E. Bullmore, Fractal connectivity of long-memory networks. Phys. Rev. E $\mathbf{7 7}(2008) 036104$.

[3] P.O. Amblard and J.F. Coeurjolly, Identification of the multivariate fractional Brownian motion. IEEE Trans. Signal Process. 59 (2011) 5152-5168.

[4] P.O. Amblard, J.F. Coeurjolly, F. Lavancier and A. Philippe, Basic properties of the multivariate fractional Brownian motion, edited by L. Chaumont. Séminaires et Congrès, Self-similar processes and their applications 28 (2012) 65-87.

[5] S. Arianos and A. Carbone, Cross-correlation of long range correlated series. J. Stat. Mech. (2009) P033037.

[6] A. Ayache, S. Leger and M. Pontier, Drap brownien fractionnaire. Potential Anal. 17 (2002) 31-43.

[7] J.M. Bardet, G. Lang, E. Moulines and P. Soulier, Wavelet estimator of long-range dependent processes. Stat. Inference Stoch. Process. 3 (2000) 85-99.

[8] G. Chan and A.T.A. Wood, Simulation of stationary Gaussian vector fields. Stat. Comput. 9 (1999) $265-268$.

[9] J.F. Coeurjolly, Estimating the parameters of a fractional Brownian motion by discrete variations of its sample paths. Stat. Inference Stoch. Process. 4 (2001) 199-227.

[10] G. Didier and V. Pipiras, Integral representations of operator fractional Brownian motions. Bernouilli 17 (2011) 1-33.

[11] G. Faÿ, E. Moulines, F. Roueff and M.S. Taqqu, Estimators of long-memory: Fourier versus wavelets. J. Econ. 151 (2009) 159-177.

[12] P. Flandrin, On the spectrum of fractional Brownian motions. IEEE Trans. Inf. Theory 35 (1988) $197-199$.

[13] P. Flandrin, Wavelet analysis and synthesis of fractional Brownian motion. IEEE Trans. Inf. Theory 38 (1992) $910-917$.

[14] I.M. Gel'fand and G.E. Shilov, Generalized functions. Properties and Operations 1 (1964).

[15] L.A. Gil-Alana, A fractional multivariate long memory model for the US and the Canadian real output. Econ. Lett. 81 (2003) $355-359$.

[16] T. Kato and E. Masry, On the spectral density of the wavelet transform of fractional Brownian motions. J. Time Ser. Anal. 20 (1999) 560-563.

[17] F. Lavancier, A. Philippe and D. Surgailis, Covariance function of vector self-similar processes. Stat. Probab. Lett. 79 (2009) 2415-2421.

[18] B. Mandelbrot and J. Van Ness, Fractional Brownian motions, fractional noises and applications. SIAM Rev. 10 (1968) 422-437.

[19] R.F. Peltier and J. Lévy-Véhel, Multifractional Brownian motion: definition and preliminary results. Rapport Recherche INRIA (1995).

[20] A.H. Tewfik and M. Kim, Correlation structure of the discrete wavelet coefficients of fractional Brownian motion. IEEE Trans. Inf. Theory 38 (1992) 904-909.

[21] D. Veitch and P. Abry, Wavelet-based joint estimate of the long-range dependence parameters. IEEE Trans. Inf. Theory 45 (1999) 878-897.

[22] B. von Bahr and C.G. Esseen, Inequalities for the $r$ th absolute moment of a sum of random variables, $1 \leq r \leq 2$. Ann. Math. Stat. 36 (1965) 299-303.

[23] G.W. Wornell, A Karhunen-Loève-like expansion for $1 / f$ processes via wavelets. IEEE Trans. Inf. Theory 36 (1990) $861-863$. 